\title{
Clinical and Radiographic Outcomes of Oblique Lumbar Interbody Fusion at 1 Year: A Preliminary Report of a Single Institution Experience
}

\author{
Doyub Kim${ }^{1}$, Byeongsam $\mathrm{Choi}^{1}$, Hae Yu Kim${ }^{1}$, Sungjoon Lee ${ }^{1,2}$ \\ ${ }^{1}$ Department of Neurosurgery, Inje University Haeundae Paik Hospital, Inje University College of Medicine, Busan, ${ }^{2}$ Department of \\ Neurosurgery, Samsung Medical Center, Sungkyunkwan University School of Medicine, Seoul, Korea
}

Corresponding Author:

Sungjoon Lee, MD

Department of Neurosurgery, Samsung

Medical Center, Sungkyunkwan

University School of Medicine, 81,

Irwon-ro, Gangnam-gu, Seoul 06351, Korea

Tel: $+82-2-3410-3499$

Fax: +82-2-3410-0048

E-mail: potata98@gmail.com

Received: February 18, 2020

Revised: March 4, 2020

Accepted: March 12, 2020

\begin{abstract}
Objective: Oblique lumbar interbody fusion (OLIF) is a minimally invasive surgical technique that is becoming popular due to lowering the risk of lumbar plexus injuries compared to other transpsoas fusion techniques while yielding comparable clinical outcomes. We evaluated the 1-year clinical and radiologic outcomes of OLIF performed in a single institution of Korea. Methods: We retrospectively reviewed patients who underwent OLIF for degenerative lumbar spinal disease at our institution between July 2015 and December 2018. Patients with a follow-up period of longer than 1 year were included. Among 36 patients, 22 patients (9 male, 13 female) and 26 surgical levels were analyzed. The patients' demographic data, surgical procedures, clinical outcomes and complications were reviewed. Results: The patients' mean age was 64 years (range 44-78). Spinal stenosis was the most common pathology. In all cases, the demineralized bone matrix was used for fusion. The disc height and sagittal angle of the index level showed statistically significant increases at the 1-year follow-up $(3.5 \mathrm{~mm}$, $\mathrm{p}<0.001 ; 5^{\circ}, \mathrm{p}=0.02$, respectively), but the foraminal height did not. The 1 -year fusion rate was $69.6 \%$. Good clinical outcomes, as evaluated by visual analogue scale pain scores, Oswestry disability index, EQ-5D-3L index and EQ-VAS, were observed throughout the 1-year followup period. No serious complications were observed. Conclusion: OLIF appears to be a suitable and safe surgical option for treating degenerative lumbar spinal disease. It showed good clinical outcomes in the short-term follow-up. However, close-long term observations would be necessary since the fusion rates were unsatisfactory.
\end{abstract}

Key Words: Lumbar vertebrae, Degenerative, Surgery, Outcome, Oblique lumbar interbody fusion

\section{INTRODUCTION}

After Ozgur et al.'s first technical report of extreme lateral interbody fusion (XLIF) was published in 2006 ${ }^{18)}$, lateral lumbar interbody fusion (LLIF) became a popular minimally invasive lumbar fusion technique and is widely performed worldwide. As surgical experience with this method has accumulated, there have been more concerns about the risk of injuring the lumbar plexus during the surgical procedures ${ }^{7,10)}$. Oblique lumbar interbody fusion (OLIF), which involves an oblique trajectory, was implemented to overcome this shortcoming of LLIF $^{8,22,23)}$. This surgical modification of LLIF has been shown to be as effective and yield similar outcomes to LLIF in multiple studies ${ }^{8,11,13,14,23)}$. However, there had been limited number of studies reporting more than 1-year long-term clinical and radiologic outcomes, especially among the Korean population. The aim of this study was to report the 1-year clinical and radiologic outcomes of OLIF in a single institution of Korea.

\section{MATERIALS AND METHODS}

Between July 2015 and December 2018, 36 patients who received OLIF surgery for degenerative lumbar spinal disease by two neurosurgeons at our institution were retrospectively reviewed after institutional review board approval was obtained (2019-10-028). We included patients who were followed-up for at least 1 year and whose medical records and radiographic images were available during the follow-up period.

The medical records were reviewed to retrieve patient in- 
formation, such as the age, sex, preoperative diagnosis, and medical histories, which included previous spine surgeries, the locations of the surgery levels, the number of operated levels, details on the surgical procedures, the operation time, and the postoperative complications. Preoperative dual-energy X-ray absorptiometry scans were performed for every female patient older than 65 years, male patient older than 70 years, and patient suspected to have secondary osteoporosis for osteoporosis evaluations. For the clinical assessments, back and leg pain scores by the visual analogue scale (VAS), Oswestry disability index (ODI), EQ-5D-3L index, and EQ-5D VAS were recorded preoperatively and at the postoperative $3-, 6^{-}$, and 12-month follow-up periods. The EQ-5D-3L index was calculated according to the equation suggested by Lee et al. for a Korean population ${ }^{12}$. In addition, surgical outcomes at 1 year were evaluated using Macnab criteria, and a descriptive analysis of the EQ-5D-3L questionnaire was performed according to Devlin et al.'s work ${ }^{4)}$.

The surgical procedures were performed as described by Woods et al. $^{23)}$. All OLIF procedures were performed initially through the left retroperitoneal space, followed by posterior percutaneous pedicle screw fixation. In cases in which the exploration of the spinal canal or direct posterior decompression was needed, laminectomy with or without facetectomy was performed additionally prior to screw fixation. For cases requiring surgery on levels L2-5, a Clydesdale cage (Medtronic, TN, USA) was used, and for cases requiring surgery on level L5-S1, a perimeter cage (Medtronic, TN, USA) was used. A demineralized bone matrix (Grafton ${ }^{\circledR}$, Medtronic, TN, USA) was filled in the cage for fusion. No intraoperative neurophysiologic monitoring was performed during the operation.

Radiologic parameters, including the index level of the average disc and foraminal heights, coronal and sagittal angles, and L1-S1 coronal and sagittal angles, were measured preoperatively, postoperative within a week, and at the 3-, 6-, and 12-month follow-ups with standing lumbar spine $X$-rays (Fig. 1) ${ }^{1)}$. The presence of endplate breech was assessed with immediate postoperative $X$-rays. Cage subsidence was identified with 12-month follow-up X-rays and graded according to the subsidence grading system suggested by Sharma et al. (Table 1$)^{21)}$. The fusion rates were evaluated based on the computed tomography (CT) scans at 12 months. Successful fusion was defined when bridging bone between the vertebral bodies and the cage was detected on a minimum of 2 contiguous sections and in at least 2 of 3 planes (axial, sagittal, or coronal) ${ }^{1)}$.

Table 1. Subsidence grading criteria

\begin{tabular}{cl}
\hline \hline Grade & Criteria description \\
\hline 0 & Normal endplate without fracture \\
I & A breach of the end plate at one side (anterior or posterior) \\
II & A fracture of the end plate at both the anterior and posterior sides of the cage \\
III & An end plate fracture with cage subsidence of more than $1 / 3$ of the cage height in to the vertebral body \\
\hline
\end{tabular}
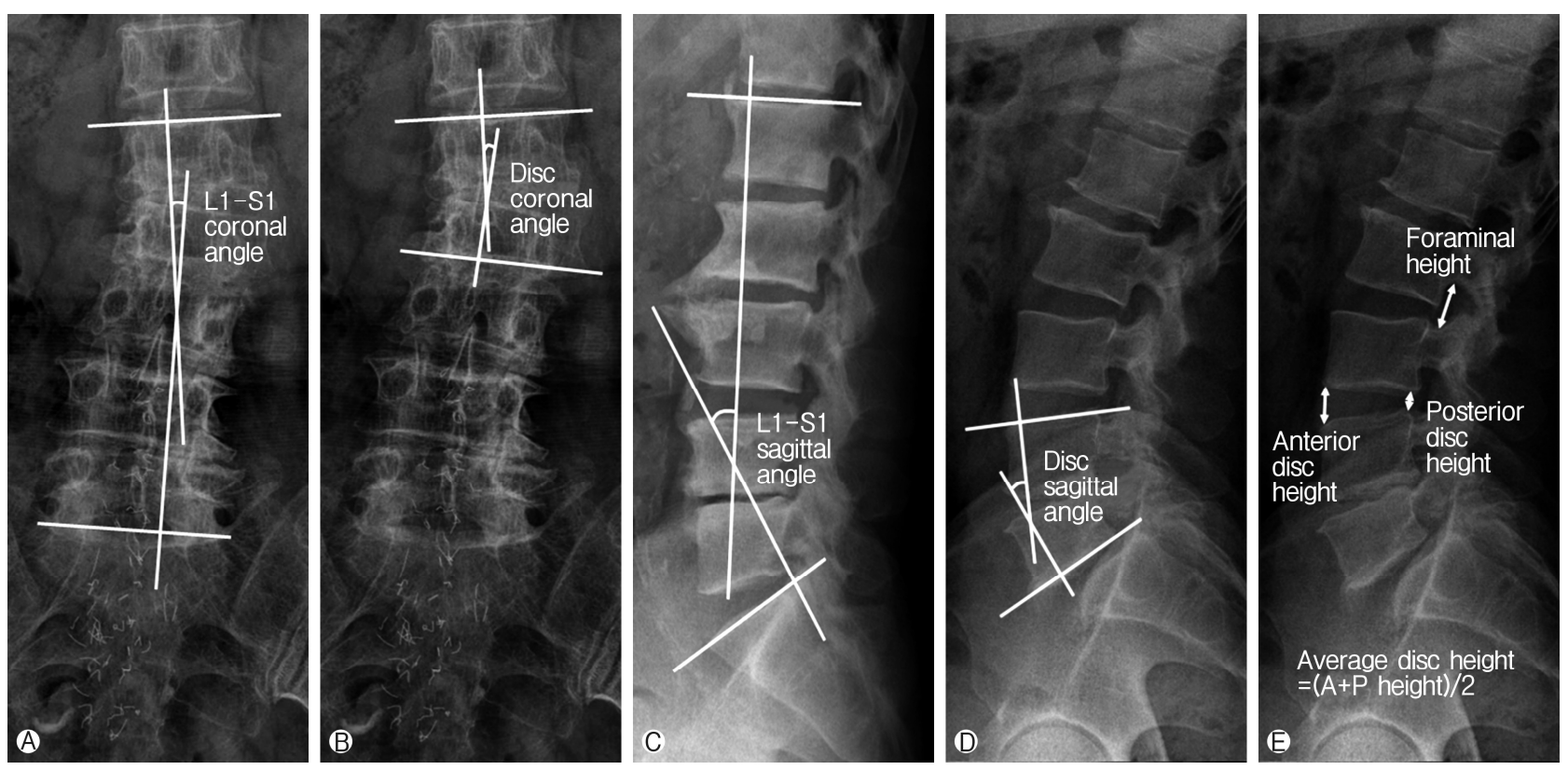

Fig. 1. Methods for measuring the radiologic parameters. (A) L1-S1 coronal Cobb angle. (B) Disc coronal angle of the index level. (C) L1-S1 sagittal lordotic angle. (D) Disc sagittal angle of the index level. (E) Average disc and foraminal heights of the index level. 


\section{Statistical Analysis}

Performed to evaluate how each radiologic parameter changed immediately after OLIF surgery and throughout the follow-up periods over 1 year. The postoperative values of each clinical assessment and radiologic parameter were compared with the preoperative values using the $t$-test. When the distribution of a variable did not follow a normal distribution, the Mann-Whitney $\mathrm{U}$ test was used. The significance level was set at $p<0.05$. All analyses were performed using MedCalc statistical software

Table 2. Demographics $(n=22)$

\begin{tabular}{|c|c|}
\hline Characteristics & Variables \\
\hline Age (years) & Mean: 64 (range: 44-78) \\
\hline \multirow[t]{2}{*}{ Gender (n) } & M: 9 \\
\hline & $\mathrm{F}: 13$ \\
\hline \multirow[t]{4}{*}{ Diagnosis (n, \%) } & Spinal stenosis $(13,59 \%)$ \\
\hline & Spondylolisthesis (7, 31\%) \\
\hline & HIVD, recur $(1,5 \%)$ \\
\hline & Instability after surgery $(1,5 \%)$ \\
\hline \multirow[t]{2}{*}{ History of spine surgery (n, \%) } & Yes $(3,14 \%)$ \\
\hline & No $(19,86 \%)$ \\
\hline \multirow[t]{3}{*}{ Osteoporosis (n, \%) } & Yes $(6,27 \%)$ \\
\hline & No $(6,27 \%)$ \\
\hline & Not evaluated $(10,46 \%)$ \\
\hline \multirow[t]{2}{*}{ Number of surgery levels ( $n, \%)$} & $1(18,82 \%)$ \\
\hline & $2(4,18 \%)$ \\
\hline \multirow[t]{3}{*}{ Surgery level $(n, \%)^{*}$} & L3-4 (6, 23\%) \\
\hline & $L 4-5(18,69 \%)$ \\
\hline & L5-S1 (2, 8\%) \\
\hline \multirow[t]{2}{*}{ Posterior decompression (n, \%) } & Yes $(5,23 \%)$ \\
\hline & No $(17,77 \%)$ \\
\hline Operative time (hours) & Mean: 3.1 (range: 1.6-5.3) \\
\hline
\end{tabular}

HIVD: herniated intervertebral disc.

*Analyzed total number of surgery levels was 26 levels. version 19.0.7 for Windows (MedCalc Software bvba, Ostend, Belgium), and RStudio software version 1.1.463 for Windows (RStudio, Inc., Boston, USA).

\section{RESULTS}

A total of 22 patients were eligible for inclusion in this study. The male to female ratio was 9:13, and the mean age was 64 years (range, 44-78). A total of 26 surgical levels were analyzed. The demographics of the study population are shown in Table 2. Posterior decompression was performed in 5 patients; 4 patients were suspected of having inadequate decompression, and 1 patient underwent the removal of a retained ruptured disc in the spinal canal.

The radiologic outcomes are summarized in Tables 3 and 4. The global sagittal and coronal angles from L1 to S1 and the segmental coronal angle were not significantly different before and after the operation. The segmental sagittal angle of the index level and the average disc and foraminal height were significantly increased after the operation and remained consistent throughout the follow-up period. However, the difference in the foraminal height was no longer statistically significant at the postoperative 1-year follow-up. The subsidence rate at 1 year was $53.8 \%$, and significant grade III subsidence, which might affect clinical outcomes negatively, were found in $8 \%$ of surgical levels. The subsidence rates were not significantly different between the patients with and without osteoporosis ( $66 \%$ vs. $50 \%, p=0.64)$, and between the male and female patients ( $30 \%$ vs. $68 \%, p=0.11$ ). The fusion rate at 1 year was $69.6 \%$ (16/23 levels, CT unavailable for 3 patients).

The clinical outcomes were mostly favorable (Table 5). The VAS scores for back and leg pain and ODI showed statistically significant decreases after the operation and remained consistent throughout the 1-year follow-up period. The EQ-5D-3L indexes and EQ-VAS scores also showed statistically significant increases after the operation and remained consistent throughout the follow-up period. When we descriptively analyzed the EQ-5D-3L questionnaire and compared the results of the preand postoperative 1-year follow-up periods (Table 6), none of the 5 dimensions showed any aggravation in the outcomes.

Table 3. Radiologic outcome ( $n=26$ levels)

\begin{tabular}{lccccc}
\hline \hline & Preop & Postop & POD 3 m & POD 6 m & POD 1 Y \\
\hline Height $(\mathrm{mm})$ & & & & & \\
$\quad$ Average disc & $8.8 \pm 3.1$ & $13.3 \pm 1.8(p<0.001)$ & $12.7 \pm 1.6(p<0.001)$ & $12.9 \pm 2.1(p<0.001)$ & $12.3 \pm 2.1(p<0.001)$ \\
$\quad$ Foraminal & $17.8 \pm 3.5$ & $20.4 \pm 6.2(p=0.002)^{*}$ & $21.1 \pm 3.1(p<0.001)$ & $19.9 \pm 3.9(p=0.04)$ & $19.7 \pm 4.1(p=0.07)$ \\
Angle $\left(^{\circ}\right)$ & & & & \\
Disc level (coronal) & $3.3 \pm 3.4$ & $2.5 \pm 2.1(p=0.653)^{*}$ & $2.6 \pm 2.4(p=0.627)^{*}$ & $2.4 \pm 2.6(p=0.272)^{*}$ & $2.6 \pm 2.2(p=0.647)^{*}$ \\
Disc level (sagittal) & $8.7 \pm 8.9$ & $15.9 \pm 7.3(p=0.003)$ & $14.0 \pm 6.5(p=0.002)$ & $14.7 \pm 6.5(p=0.008)$ & $13.7 \pm 7.1(p=0.029)$ \\
L1-S1 (coronal) & $3.3 \pm 2.4$ & $3.8 \pm 2.9(p=0.733)^{*}$ & $3.6 \pm 3.9(p=0.562)^{*}$ & $3.6 \pm 3.7(p=0.934)^{*}$ & $3.6 \pm 2.8(p=0.673)$ \\
L1-S1 (sagittal) & $36.3 \pm 13.4$ & $38.5 \pm 11.5(p=0.565)$ & $44.7 \pm 12.9(p=0.02)^{*}$ & $41.6 \pm 14.9(p=0.221)$ & $39.1 \pm 12.0(p=0.469)$ \\
\hline
\end{tabular}

Preop: preoperative; POD: postoperative days; Postop: postoperative; $3 \mathrm{~m}=3$ months; $6 \mathrm{~m}=6$ months; $1 \mathrm{Y}=1$ year.

"Man-Whitney U test was used. 


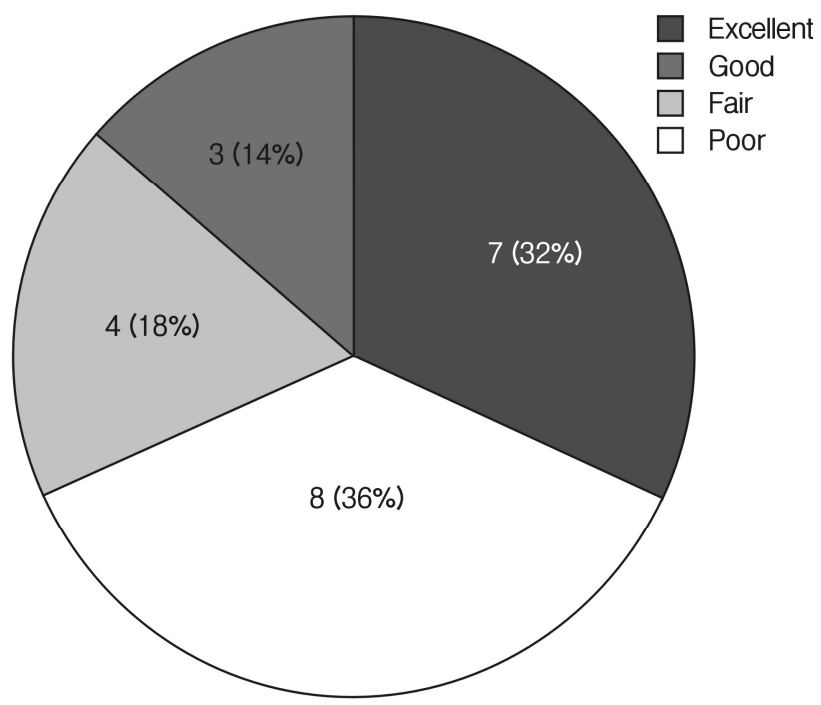

Fig. 2. Clinical outcome evaluation performed according to the Macnab criteria at the postoperative 1-year follow-up.
It is notable that the usual activities dimension improved the most and the pain/discomfort dimension improved the least. According to the Macnab criteria, approximately $86 \%$ of the patients showed better than fair clinical outcomes at the postoperative 1-year follow-up (Fig. 2).

The complications are summarized in Table 7. There were no serious complications related to the operation. Transient left anterior hip flexor grade 4+ weakness and left anterior thigh sensory change were observed in $2(9 \%)$ and $1(5 \%)$ patients, respectively. Unexplainable right knee extensor grade 4 weakness was observed in 1 patient. All 4 patients completely recovered by the 3-month follow-up.

There was 1 patient who required a revision surgery. Her initial symptoms, which were pain in the back and left leg and grade 4 motor weakness in ankle dorsiflexion, improved somewhat after the initial operation (Fig. 3A-D). However, she complained that the amount of pain relief was not satisfactory. In addition, the motor weakness status remained similar to the preoperative status. At 6 months postoperatively, we performed additive posterior decompression surgery (Fig. 3E-G). The leg
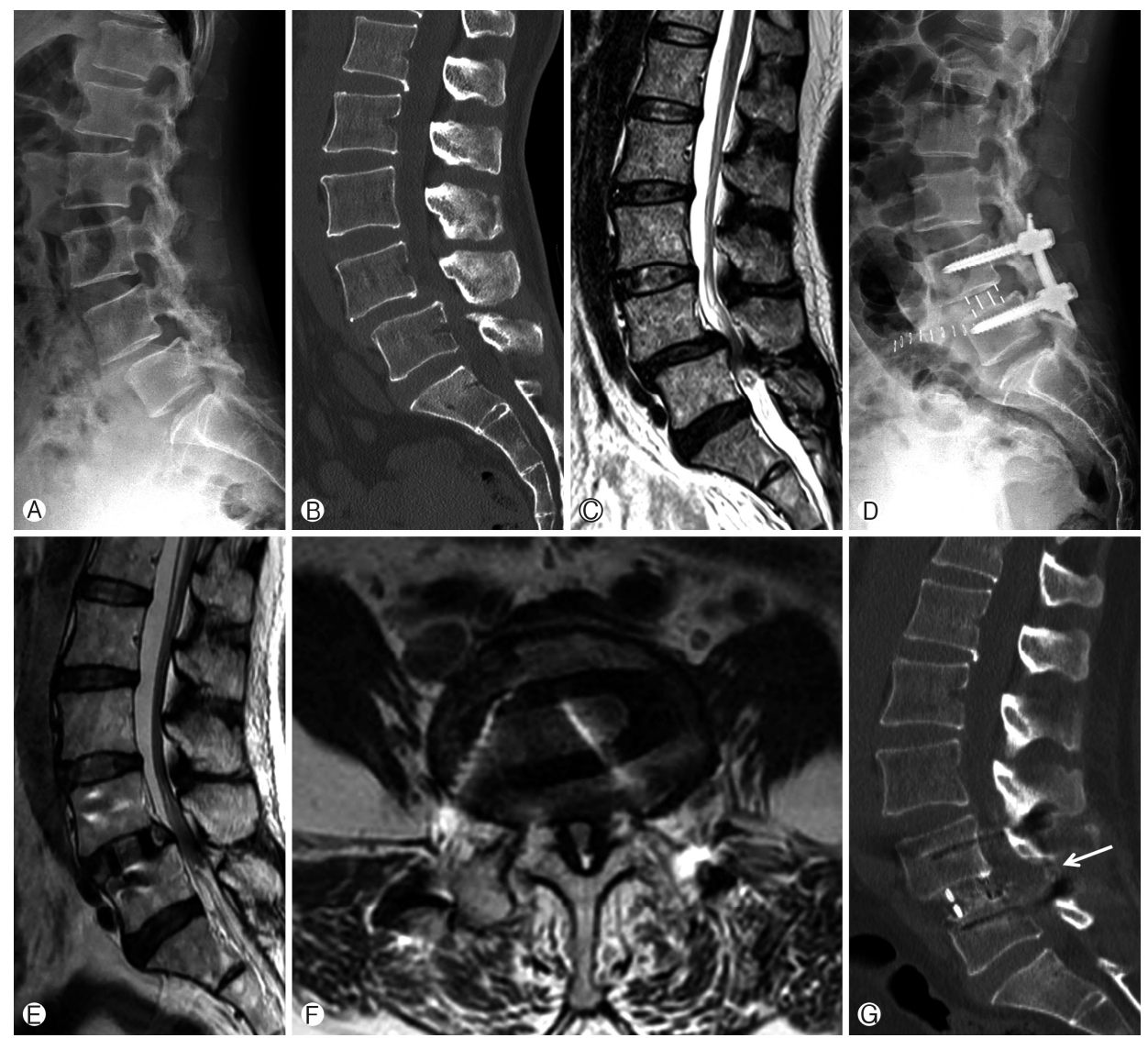

Fig. 3. Preoperative (A) simple lateral radiograph, (B) mid-sagittal view of CT, (C) T2-weighted sagittal MRI of the lumbar spine. Spondylolisthesis with severe spinal canal stenosis at L4-5 are shown. (D) Immediate postoperative simple lateral radiograph. Improved disc height, but partial reduction of the L4-5 spondylolisthesis was noted. Postoperative 6-month follow-up (E) T2-weighted sagittal, and (F) axial MRI showed still remaining stenosis. (G) CT sagittal image taken after the second operation. Partial laminectomy site was seen (white arrow). 
pain was reduced by an additional 50\%. However, her left ankle motor weakness did not improve even after the $2^{\text {nd }}$ surgery.

\section{DISCUSSION}

Lumbar interbody fusion is an established treatment option for degenerative lumbar spinal diseases. Four main approaches, which include posterior, transforaminal, anterior and lateral lumbar interbody fusion, are widely used. Currently, there is no definite evidence of one approach being superior to another in terms of the fusion rate or clinical outcomes ${ }^{17)}$. However, each approach has its own clear advantages and disadvantages. LLIF shares the advantages of other minimally invasive techniques, such as reduced blood loss, a fast recovery, and decreased postoperative pain, which enables early postoperative mobilization for patients ${ }^{8}$. In addition, its advantages include easy access to multiple lumbar levels, a short operation time, minimizing posterior musculature injury, and ability to correct coronal and sagittal deformities and restore the disc height ${ }^{8,17)}$. Despite all these advantages, LLIF also has unique shortcomings. Concerns regarding its approach-related complications, such as sensorimotor deficits of the thigh on the approached side caused by injuries to the psoas muscle or the lumbar plexus, have been most commonly described ${ }^{6,8,17)}$. Damage to intraabdominal organs and major vessels can also occur. OLIF seems to inherit these characteristics of LLIF and yield similar clinical and radiologic outcomes ${ }^{11,14,16,23)}$. There are concerns of a high risk of injuring intraabdominal organs, sympathetic chains and major vessels. However, both approaches showed similarly low rates

Table 4. Subsidence and fusion rate at postoperative 1-year ( $n=26$ levels)

\begin{tabular}{lc}
\hline \hline Variables & Numbers (\%) \\
\hline Endplate breech at postop & $2(7.7 \%)$ \\
Subsidence at 1-year & $14(53.8 \%)$ \\
Subsidence grade & \\
0 & $12(46 \%)$ \\
I & $4(15 \%)$ \\
II & $8(31 \%)$ \\
III & $2(8 \%)$ \\
Fusion at 1-year & $16(70 \%)$ \\
\hline
\end{tabular}

${ }^{*} \mathrm{CT}$ at postoperative 1-year was not available in 3 patients. of such complications ${ }^{8,9,11,14,23)}$. Moreover, OLIF appears to have a significant advantage in preventing sensorimotor deficits of the thigh compared to LLIF, ${ }^{8,11,16)}$. Considering that OLIF enables the approach to L5-S1, which is not possible with LLIF ${ }^{16)}$, OLIF appears to have advantages over LLIF in some aspects.

The postoperative 1-year clinical outcomes reported in this study appear to be acceptable compared with the historical data from many studies covering various lumbar fusion techniques $^{3,19)}$. The back and leg VAS scores and ODI scores significantly decreased from the preoperative values throughout the 1-year follow-up period (Table 5). In addition, the EQ-5D-3L indexes and EQ-VAS scores significantly increased postoperatively and remained consistent for one year. No specific quality of life dimension score deteriorated 1-year postoperatively compared with preoperatively (Table 6).

Decompressing the neural structures in an indirect fashion has proven to be effective in treating patients with degenerative lumbar spinal stenosis in several studies $5,15,19,20)$. Our study also showed similar results. There were 5 cases that we added posterior decompression. There was one case in which we explored the spinal canal to remove sequestrated disc material, but it remains unclear whether the posterior decompression procedure was truly a necessary procedure in the other 4 cases. However, there was one revision case in which we performed additional posterior decompression 6 months after the initial OLIF surgery. In this case, the spondylolisthesis of the index level was not sufficiently corrected, and the patient experienced some improvements in her symptoms after the $2^{\text {nd }}$ surgery. This finding indicates that additionally performing a posterior decompression procedure is beneficial in some specific cases and should be considered when the sufficiency of indirect decompression is in question.

The noticeable findings in the radiologic outcomes include the low fusion rate. The estimated fusion rate at the postoperative 1 -year follow-up was approximately $70 \%$ in our study. However, in the literature, Woods et al. reported a fusion rate of $98 \%$ at 6 months following OLIF surgery ${ }^{23)}$. Although we have to be very careful in directly comparing the results of two independent retrospective studies, we presume that the most plausible explanation for this difference in the fusion rate is that the fusion material used was different. Woods et al. used bone morphogenetic proteins for fusion in their study ${ }^{23)}$, whereas we used a demineralized bone matrix only. We found one study conducted in a Korean population in the literature reporting a higher than that in our study when using a demineralized

Table 5. Clinical outcomes $(n=15)$

\begin{tabular}{lcccc}
\hline \hline Variables & Preoperative & 3-month & 6-month & 1-year \\
\hline Back VAS (range: 0-10) & $6.3 \pm 2.8$ & $3.2 \pm 2.2(p=0.003)$ & $2.4 \pm 1.8(p<0.001)$ & $3.4 \pm 1.9(p=0.005)$ \\
Leg VAS (range: 0-10) & $7.9 \pm 2.2$ & $2.6 \pm 2.3(p<0.001)$ & $2.5 \pm 1.9(p<0.001)$ & $3.0 \pm 1.9(p<0.001)$ \\
ODI (range: 0-45) & $23.1 \pm 6.2$ & $13.2 \pm 7.6(p<0.001)$ & $13.2 \pm 7.6(p<0.001)$ & $11.3 \pm 7.2(p<0.001)$ \\
EQ-5D-3L index (range: $0.95--0.171)$ & $0.55 \pm 0.151$ & $0.753 \pm 0.122(p<0.001)$ & $0.779 \pm 0.113(p<0.004)^{*}$ & $0.778 \pm 0.134(p<0.001)$ \\
EQ-VAS (range: 0-100) & $35.87 \pm 22.91$ & $65.0 \pm 14.46(p<0.001)$ & $67.31 \pm 15.49(p<0.001)$ & $67.27 \pm 21.25(p<0.002)$ \\
\hline
\end{tabular}

*Mann-Whitney $U$ test was used. 
Doyub Kim, et al.

Table 6. Numbers and proportions reporting levels within EQ-5D dimensions: Pre-operation and 1-year follow-up

\begin{tabular}{|c|c|c|c|c|c|c|c|c|c|c|}
\hline \multirow{2}{*}{ Level } & \multicolumn{2}{|c|}{ Mobility } & \multicolumn{2}{|c|}{ Self-care } & \multicolumn{2}{|c|}{ Usual activities } & \multicolumn{2}{|c|}{ Pain/discomfort } & \multicolumn{2}{|c|}{ Anxiety/depression } \\
\hline & Preop & 1-year & Preop & 1-year & Preop & 1-year & Preop & 1-year & Preop & 1-year \\
\hline 1 & $\begin{array}{c}0 \\
(0 \%)\end{array}$ & $\begin{array}{c}5 \\
(33.3 \%)\end{array}$ & $\begin{array}{c}6 \\
(40 \%)\end{array}$ & $\begin{array}{c}9 \\
(60 \%)\end{array}$ & $\begin{array}{c}0 \\
(0 \%)\end{array}$ & $\begin{array}{c}8 \\
(53.3 \%)\end{array}$ & $\begin{array}{c}0 \\
(0 \%)\end{array}$ & $\begin{array}{c}2 \\
(13.3 \%)\end{array}$ & $\begin{array}{c}2 \\
(13.3 \%)\end{array}$ & $\begin{array}{c}8 \\
(53.3 \%)\end{array}$ \\
\hline 2 & $\begin{array}{c}15 \\
(100 \%)\end{array}$ & $\begin{array}{c}10 \\
(66.7 \%)\end{array}$ & $\begin{array}{c}9 \\
(60 \%)\end{array}$ & $\begin{array}{c}6 \\
(40 \%)\end{array}$ & $\begin{array}{c}10 \\
(66.7 \%)\end{array}$ & $\begin{array}{c}6 \\
(40 \%)\end{array}$ & $\begin{array}{c}8 \\
(53.3 \%)\end{array}$ & $\begin{array}{c}13 \\
(86.7 \%)\end{array}$ & $\begin{array}{c}11 \\
(73.3 \%)\end{array}$ & $\begin{array}{c}7 \\
(46.7 \%)\end{array}$ \\
\hline 3 & $\begin{array}{c}0 \\
(0 \%)\end{array}$ & $\begin{array}{c}0 \\
(0 \%)\end{array}$ & $\begin{array}{c}0 \\
(0 \%)\end{array}$ & $\begin{array}{c}0 \\
(0 \%)\end{array}$ & $\begin{array}{c}5 \\
(33.3 \%)\end{array}$ & $\begin{array}{c}1 \\
(6.7 \%)\end{array}$ & $\begin{array}{c}7 \\
(46.7 \%)\end{array}$ & $\begin{array}{c}0 \\
(0 \%)\end{array}$ & $\begin{array}{c}2 \\
(13.3 \%)\end{array}$ & $\begin{array}{c}0 \\
(0 \%)\end{array}$ \\
\hline Total & $\begin{array}{c}15 \\
(100 \%)\end{array}$ & $\begin{array}{c}15 \\
(100 \%)\end{array}$ & $\begin{array}{c}15 \\
(100 \%)\end{array}$ & $\begin{array}{c}15 \\
(100 \%)\end{array}$ & $\begin{array}{c}15 \\
(100 \%)\end{array}$ & $\begin{array}{c}15 \\
(100 \%)\end{array}$ & $\begin{array}{c}15 \\
(100 \%)\end{array}$ & $\begin{array}{c}15 \\
(100 \%)\end{array}$ & $\begin{array}{c}15 \\
(100 \%)\end{array}$ & $\begin{array}{c}15 \\
(100 \%)\end{array}$ \\
\hline Number reporting some problems ${ }^{*}$ & $\begin{array}{c}15 \\
(100 \%)\end{array}$ & $\begin{array}{c}10 \\
(66.7 \%)\end{array}$ & $\begin{array}{c}9 \\
(60 \%)\end{array}$ & $\begin{array}{c}6 \\
(40 \%)\end{array}$ & $\begin{array}{c}15 \\
(100 \%)\end{array}$ & $\begin{array}{c}7 \\
(46.7 \%)\end{array}$ & $\begin{array}{c}15 \\
(100 \%)\end{array}$ & $\begin{array}{c}13 \\
(86.7 \%)\end{array}$ & $\begin{array}{c}13 \\
(86.7 \%)\end{array}$ & $\begin{array}{c}7 \\
(46.7 \%)\end{array}$ \\
\hline Change in numbers reporting problems & \multicolumn{2}{|c|}{-5} & \multicolumn{2}{|c|}{-3} & \multicolumn{2}{|c|}{-8} & \multicolumn{2}{|c|}{-2} & \multicolumn{2}{|c|}{-6} \\
\hline$\%$ change in numbers reporting problems & \multicolumn{2}{|c|}{$-33.3 \%$} & \multicolumn{2}{|c|}{$-20 \%$} & \multicolumn{2}{|c|}{$-53.3 \%$} & \multicolumn{2}{|c|}{$-13.3 \%$} & \multicolumn{2}{|c|}{$-40 \%$} \\
\hline Rank of dimensions in terms of $\%$ changes & \multicolumn{2}{|c|}{3} & \multicolumn{2}{|c|}{4} & \multicolumn{2}{|c|}{1} & \multicolumn{2}{|c|}{5} & \multicolumn{2}{|c|}{2} \\
\hline
\end{tabular}

"'Some problems'=level $2+3$

Table 7. Complications $(\mathrm{n}=22)$

\begin{tabular}{lc}
\hline \hline Complications & Numbers (\%) \\
\hline Left anterior hip flexor transient weakness & $2(9 \%)^{*}$ \\
Left anterior thigh transient sensory change & $1(5 \%)^{*}$ \\
Neurologic injury & \\
- Right quadriceps weakness & $1(5 \%)^{*}$ \\
Revision surgery & $1(5 \%)$ \\
Postoperative ileus & $1(5 \%)$ \\
Vascular or ureter injury & $0(0 \%)$ \\
Retrograde ejaculation & $0(0 \%)$ \\
Wound infection & $0(0 \%)$ \\
Blood transfusion & $0(0 \%)$ \\
\hline
\end{tabular}

${ }^{*}$ All symptoms were completely resolved at postoperative 3 months follow-up.

bone matrix in OLIF surgery. Lin et al. reported a fusion rate of approximately $88 \%$ with the use of demineralized bone matrix only at 1 year after OLIF surgery ${ }^{11)}$. However, this was a small number, matched-pair comparison study that the authors did not include their whole patient series. Therefore, hidden bias may be present in the study, and we should interpret the results with caution.

A delay in fusion seems to affect other radiologic parameters, such as the subsidence rate and disc and foraminal heights. Although most of the subsidence cases that occurred were minor (subsidence grade II or lower), it was observed in over $50 \%$ of the patients (Table 4). Although patients with osteoporosis seemed to be prone to subsidence (66\% vs. $50 \%)$, the difference was not statistically significant. Notably, $50 \%$ of patients without evidence of osteoporosis had experienced subsidence to least a minimal degree. Compared with the preoperative values, the average disc height gradually decreased, and the mean value of the foraminal height was no longer significant at the 1-year follow-up (Table 3). Fortunately, the clinical outcomes did not show any signs of deterioration. However, it is worrisome that a gradual decrease in the indirect decompression effect might lead to clinical deterioration in the long term. The use of a larger cage, positioning the cage on both sides of the hard cortical vertebral rim and saving the posterior spinal structures are thought to be key features of LLIF and OLIF which provide more resistance to subsidence and great ability to correct deformity compared with other lumbar fusion techniques ${ }^{16-18)}$. It has been suggested that these features allowed us more freedom in selecting fusion materials. However, our data indicates that this may not true. Although it is too early to determine the adequacy of use of a demineralized bone matrix and more long-term follow-up data is required for a proper evaluation, our preliminary data suggest that seeking an alternative strategy rather than using a demineralized bone matrix alone as the fusion material is necessary.

Approach-related complications, especially left thigh motor and sensory deficits, were observed in 3 of 22 patients, and all of the complications completely resolved by the 3-month postoperative follow-up. As several studies have reported, we think that OLIF can be safely performed without intraoperative neurophysiologic monitoring ${ }^{8,14,23)}$. We think that manipulating the psoas muscle during the surgery might have caused transient hip flexor weakness which was persisted only immediate postoperative period. Also, we presume that a left anterior thigh sensory change happened in one patient might be caused by manipulation or retraction of the genitofemoral nerve. No grave complications, such as large vessel injuries, occurred in our series. However, there have been several reports concerning intraoperative venous injuries ${ }^{2,8,16)}$, and surgeons must be careful in presurgical planning and open-minded in modifying the plan if radiographic vessel studies suggest a high risk of vessel injuries, especially at the L5-S1 level.

This study has several limitations. First, it is a retrospective, single-arm study without a control group for comparison. 
Second, we could only include approximately $60 \%$ of the patients in the study, and it is possible that the results from this study may not represent the whole patient group. Third, the number of patients used in the clinical data analysis was smaller than the number of patients used in the radiologic data analysis. Therefore, it is possible that the evidence of deterioration shown in the radiographic parameters, such as the decrease in the foraminal height and low fusion rates, might not be properly reflected in the clinical outcomes. Fourth, the small number of study population limited overall credibility of the study results. In addition, this had limited thorough analysis of known meaningful factors, such as gender, osteoporosis, and several technical aspects of OLIF surgery. Lastly, this is a preliminary result of a single center for only a 1-year follow-up period. For a proper evaluation of OLIF outcomes in treating lumbar degenerative spine diseases, more long-term follow-up data should be acquired though future studies.

\section{CONCLUSION}

Oblique lumbar interbody fusion is a safe surgical procedure for treating degenerative lumbar spinal diseases. It yielded good clinical outcomes at the 1-year follow-up. The fusion rate at 1 year was approximately $70 \%$ when only a demineralized bone matrix was used. Close long-term follow-up would be necessary. If deteriorations of the clinical outcomes observed in longer follow-ups, then we may need an alternative strategy to achieve earlier fusion.

\section{REFERENCES}

1. Alimi M, Hofstetter CP, Cong GT, Tsiouris AJ, James AR, Paulo D, et al: Radiological and clinical outcomes following extreme lateral interbody fusion. J Neurosurg Spine 20:623-635, 2014

2. Assina R, Majmundar NJ, Herschman Y, Heary RF: First report of major vascular injury due to lateral transpsoas approach leading to fatality. J Neurosurg Spine 21:794-798, 2014

3. de Kunder SL, van Kuijk SMJ, Rijkers K, Caelers I, van Hemert WLW, de Bie RA, et al: Transforaminal lumbar interbody fusion (TLIF) versus posterior lumbar interbody fusion (PLIF) in lumbar spondylolisthesis: a systematic review and meta-analysis. Spine J 17:1712-1721, 2017

4. Devlin NJ, Parkin D, Browne J: Patient-reported outcome measures in the NHS: new methods for analysing and reporting EQ-5D data. Health Econ 19:886-905, 2010

5. Fujibayashi S, Hynes RA, Otsuki B, Kimura H, Takemoto M, Matsuda S: Effect of indirect neural decompression through oblique lateral interbody fusion for degenerative lumbar disease. Spine (Phila Pa 1976) 40:E175-182, 2015

6. Goyal A, Kerezoudis P, Alvi MA, Goncalves S, Bydon M: Outcomes following minimally invasive lateral transpsoas interbody fusion for degenerative low grade lumbar spondylolisthesis: A systematic review. Clin Neurol Neurosurg 167:122-128, 2018

7. Graham RB, Wong AP, Liu JC: Minimally invasive lateral transpsoas approach to the lumbar spine: pitfalls and complication avoidance. Neurosurg Clin N Am 25:219-231, 2014

8. Hah R, Kang HP: Lateral and oblique lumbar interbody fu- sion-current concepts and a review of recent literature. Curr Rev Musculoskelet Med 305-310, 2019

9. Hijji FY, Narain AS, Bohl DD, Ahn J, Long WW, DiBattista JV, et al: Lateral lumbar interbody fusion: a systematic review of complication rates. Spine J 17:1412-1419, 2017

10. Houten JK, Alexandre LC, Nasser R, Wollowick AL: Nerve injury during the transpsoas approach for lumbar fusion. $\mathrm{J}$ Neurosurg Spine 15:280-284, 2011

11. Jin J, Ryu KS, Hur JW, Seong JH, Kim JS, Cho HJ: Comparative study of the difference of perioperative complication and radiologic results: MIS-DLIF (Minimally Invasive Direct Lateral Lumbar Interbody Fusion) versus MIS-OLIF (Minimally Invasive Oblique Lateral Lumbar Interbody Fusion). Clin Spine Surg 31:31-36, 2018

12. Lee YK, Nam HS, Chuang LH, Kim KY, Yang HK, Kwon IS, et al: South Korean time trade off values for EQ 5D health states: Modeling with observed values for 101 health states. Value in Health 12:1187-1193, 2009

13. Li HM, Zhang RJ, Shen CL: Radiographic and clinical outcomes of oblique lateral interbody fusion versus minimally invasive transforaminal lumbar interbody fusion for degenerative lumbar disease. World Neurosurg 122:e627-e638, 2019

14. Li JX, Phan K, Mobbs R: Oblique lumbar interbody fusion: technical aspects, operative outcomes, and complications. World Neurosurg 98:113-123, 2017

15. Lin GX, Akbary K, Kotheeranurak V, Quillo-Olvera J, Jo HJ, Yang XW, et al: Clinical and radiologic outcomes of direct versus indirect decompression with lumbar interbody fusion: A matched-pair comparison analysis. World Neurosurg 119: e898-e909, 2018

16. Miscusi M, Ramieri A, Forcato S, Giuffre M, Trungu S, Cimatti $\mathrm{M}$, et al: Comparison of pure lateral and oblique lateral interbody fusion for treatment of lumbar degenerative disk disease: a multicentric cohort study. Eur Spine J 27:222-228, 2018

17. Mobbs RJ, Phan K, Malham G, Seex K, Rao PJ: Lumbar interbody fusion: techniques, indications and comparison of interbody fusion options including PLIF, TLIF, MI-TLIF, OLIF/ATP, LLIF and ALIF. J Spine Surg 1:2-18, 2015

18. Ozgur BM, Aryan HE, Pimenta L, Taylor WR: Extreme Lateral Interbody Fusion (XIIF): a novel surgical technique for anterior lumbar interbody fusion. Spine J 6:435-443, 2006

19. Phan K, Thayaparan GK, Mobbs RJ: Anterior lumbar interbody fusion versus transforaminal lumbar interbody fusion--systematic review and meta-analysis. Br J Neurosurg 29:705-711, 2015

20. Rao PJ, Maharaj MM, Phan K, Lakshan Abeygunasekara M, Mobbs RJ: Indirect foraminal decompression after anterior lumbar interbody fusion: a prospective radiographic study using a new pedicle-to-pedicle technique. Spine J 15:817-824, 2015

21. Sharma AK, Kepler CK, Girardi FP, Cammisa FP, Huang RC, Sama AA: Lateral lumbar interbody fusion: clinical and radiographic outcomes at 1 year: a preliminary report. J Spinal Disord Tech 24:242-250, 2011

22. Silvestre C, Mac-Thiong JM, Hilmi R, Roussouly P: Complications and morbidities of mini-open anterior retroperitoneal lumbar interbody fusion: Oblique lumbar interbody fusion in 179 patients. Asian Spine J 6:89-97, 2012

23. Woods KR, Billys JB, Hynes RA: Technical description of oblique lateral interbody fusion at L1-L5 (OLIF25) and at L5-S1 (OLIF51) and evaluation of complication and fusion rates. Spine J 17:545-553, 2017 